\title{
Zur Relevanz verschiedener Lebensbereiche für die Entstehung individueller Werthaltungen. Aktuelle Befunde für Österreich aus einer Mixed-Methods-Studie
}

\author{
Roland Verwiebe $\cdot$ Lena Seewann • Margarita Wolf
}

(C) Der/die Autor(en) 2018

Zusammenfassung Dieser Beitrag thematisiert Werthaltungen und fragt danach, wo und wie sie sich herausbilden. Basis ist ein österreichisches Projekt mit MixedMethods-Ansatz, in dem wertebildende Lebensbereiche in den Blick genommen wurden. Wir nutzen Daten aus acht Fokusgruppen und einen repräsentativen Survey mit 1519 ÖsterreicherInnen. Bisherige empirische Studien zeigen, dass die Entstehung individueller Werthaltungen hauptsächlich durch familiale und pädagogische Vermittlungsprozesse vonstattengeht. Die vorliegenden Analysen machen jedoch deutlich, dass eine Reihe weiterer Lebensbereiche für die Entstehung von Werten entscheidend ist. Wertebildung geht häufig mit besonderen individuellen Erfahrungen einher, die nicht nur positive Erlebnisse, sondern auch Konflikt- und Krisensituationen umfassen. Unsere Befunde zeigen zudem, dass dieser komplexe Prozess von verschiedenen sozialen Gruppen sehr unterschiedlich bewertet wird, was bisher selten empirisch aufgearbeitet wurde.

Schlüsselwörter Werte $\cdot$ Wertebildung Österreich $\cdot$ Mixed-Methods-Studie

\section{The relevance of different areas of life for the formation of individual values. Recent mixed-methods findings for Austria.}

\begin{abstract}
This article explores the individual values and asks where and how they are formed. A current research project in Austria forms the basis for analysis, employing a mixed-methods design to focus on processes that develop and influence values. We use data from eight focus group discussions and a representative survey of 1519 Austrians. Previous empirical studies locate the formation of individual values in familial or educational processes. However, the present analysis shows that
\end{abstract}

R. Verwiebe $(\bowtie) \cdot$ L. Seewann $\cdot$ M. Wolf Institut für Soziologie, Universität Wien, Rooseveltplatz 2, 1090 Wien, Österreich

E-Mail: roland.verwiebe@univie.ac.at 
a multitude of other areas of life play an equally important role in the emergence of individual value systems. The formation of values is linked to exceptional, individual experiences that are not inclusively positive, but often tied to conflicts or situations of crisis. The results further highlight, that this complex process is perceived quite differently among diverse social groups; that also has been only seldomly addressed by existing empirical research.

Keywords Values $\cdot$ Formation of values - Austria $\cdot$ Mixed methods study

\section{Einleitung}

Was sind Werte? Welche Werte haben Menschen, die in Österreich leben, und welche sind für sie besonders wichtig? Wie entstehen individuelle Werthaltungen und wie stabil sind diese? Dies sind Fragen, die besonders seit dem Beginn der humanitären Krise 2015 und nach der Vorstellung des Integrationsplans für Geflüchtete im November 2015 und den darin enthaltenen Orientierungs- und Wertekursen vermehrt in der Öffentlichkeit diskutiert wurden. Im Vordergrund stand dabei die Vermittlung von Werten an ZuwanderInnen, die erst kürzlich nach Österreich gekommen sind. Auch im Wahlkampf zur letzten Nationalratswahl von 2017 war das Thema Werte zentral, was sich an vielfachen Wortmeldungen aus unterschiedlichen politischen Lagern zeigte. In beiden öffentlichen Diskursen wurde immer wieder ein Mehr an Werten und orientierungsstiftenden Idealen gefordert, nach Schubarth (2010) ein symptomatischer Ausdruck einer von Krisen und Verunsicherung geprägten Gesellschaft. Dabei sind die medialen Auseinandersetzungen von teilweise zuspitzenden Äußerungen geprägt, wobei mit sehr unterschiedlichen Wertedefinitionen und Vorstellungen von Wertevermittlungsprozessen operiert wird. Die Bandbreite lässt sich anhand von Zeitungsüberschriften veranschaulichen, die von „Unsere Werte sind für Zuwanderer uninteressant“ (Krone, 15. Mai 2015), „Auf der Suche nach österreichischen Werten“ (Die Presse, 2. Februar 2015) bis zu „Ein erzwungener Wert ist niemals ein Wert“ (Standard, 28. November 2016) und „Was sind Werte wert“" (Wiener Zeitung, 19. August 2017) reichen.

Der vorliegende Beitrag verfolgt vor diesem Hintergrund das Ziel, ein Desiderat der Werteforschung aufzugreifen, indem die Entstehung individueller Werthaltungen in unterschiedlichen Lebensbereichen untersucht wird. Trotz einer steigenden Zahl von Publikationen im Bereich der Einstellungs- und Werteforschung - vor allem auf Basis standardisierter Befragungen (Thome 2003, S. 5-6) - sind Studien zu dem hier gewählten Forschungsfokus eher selten zu finden. Die empirische Basis für den vorliegenden Beitrag liefert ein aktuelles Mixed-Methods-Projekt, welches seit 2016 an der Universität Wien durchgeführt wird. Zum einen wird mittels der Befunde aus mehreren Gruppendiskussionen erörtert, welche Erfahrungen für die eigenen Werte als besonders prägend empfunden werden und wie diese Erfahrungen in verschiedenen Lebensbereichen ineinandergreifen. Zum anderen soll durch Analysen eines für Österreich repräsentativen Surveys $(N=1500)$ untersucht werden, welche Lebensbereiche für die Entstehung von individuellen Werthaltungen besonders wichtig sind und welche Unterschiede sich hinsichtlich der Wahrnehmung dieser Wertebildungs- 
prozesse aufgrund sozialstruktureller Merkmale wie Geschlecht, Alter und Bildung feststellen lassen. Trotz des Querschnittdesigns kann die Entstehung individueller Werthaltungen auf Basis unseres empirischen Materials damit aus einer bisher selten betrachteten Perspektive beleuchtet werden. Fokusgruppendiskussionen erlauben uns zunächst aufzuzeigen, wie über Werte und Wertebildung gesprochen und reflektiert wird. Einerseits finden damit Kommunikations- und Aushandlungsprozesse innerhalb des Gruppensettings Berücksichtigung (Morgan 1997). Andererseits, und darauf liegt der Schwerpunkt des Beitrages, können auch subjektive und biografische Erfahrungen auf Individualebene beachtet werden. Anschließend kann über die systematische Auswertung quantitativer Daten erörtert werden, wie sich dieser Prozess möglicherweise zwischen verschiedenen sozialen Gruppen unterscheidet. Mit Rückblick auf die qualitativen Ergebnisse wählen wir dafür einen besonderen Fokus und untersuchen erstmalig für Österreich einen besonderen Ausschnitt der Entstehung individueller Werthaltungen, nämlich die Rolle von Konflikten und negativen Prägungserfahrungen im Leben. Nimmt man beide Perspektiven zusammen, so liefern unsere Mixed-Methods-Analysen Ergebnisse zu der Entstehung individueller Werthaltungen, der Verortung von Wertebildungsprozessen in diversen Lebensbereichen, der Identifizierung gemeinsamer oder divergenter Erfahrungen in diesem Zusammenhang und der Beeinflussung dieser Erfahrungen durch sozialstrukturelle Faktoren.

\section{Theoretischer Hintergrund und Stand der Forschung}

Der Wertebegriff wird in der Alltagssprache in vielfältiger Weise verwendet. Gemeinhin bezeichnet er die Bedeutung oder Nützlichkeit einer Sache, es können aber auch Wünsche oder Erwünschtes damit gemeint sein. Häufig werden Werte somit als ein „Synonym für divergente Phänomene wie Interessen, Einstellungen, Präferenzen, Motivationen, Mittel, Ziele“ gebraucht (Polak et al. 2009, S. 22). Diese unterschiedlichen Bedeutungsebenen des Wertebegriffs kommen auch in wissenschaftlichen Diskussionen zum Tragen. In der sozialwissenschaftlichen Theorie wird Werten eine hohe Relevanz für gesellschaftlichen Zusammenhalt beigemessen. In der Philosophie und Ethik wird der Wertebegriff als Pluralisierung und Subjektivierung des „Guten“ verstanden (Joas 2006, S. 5-6), was ihn jedoch aus philosophischer Sicht in seiner Verallgemeinerbarkeit einschränkt. „Werte spielen daher zwar als Zielvorstellungen des Guten eine wichtige Rolle, müssen aber in einen ethischen Diskurs eingebettet und überprüft werden“, formuliert zum Beispiel Polak in diesem Zusammenhang (2011, S. 26). In soziologischen oder politikwissenschaftlichen Ansätzen werden Werte hingegen auf der Ebene von Ideen und Vorstellungen verortet und gelten hier als Gedankenkonstrukte bzw. kulturelle Ideen (Spates 1983; Thome 2003). In dieser Hinsicht sind sie abstrakt, generalisiert und auch nicht eins zu eins in eine Handlung übertragbar. Damit sich Werte auf unser Verhalten auswirken können, müssen sie je nach Situation erst in soziale Normen ,übersetzt“ werden. Sozialpsychologische Ansätze gehen außerdem häufig von der Existenz universell geltender Grundwerte aus (Schwartz 1994), wie etwa Sicherheit, Tradition, Hedonismus oder Universalismus. 
Im Unterschied zur theoretischen Debatte um den Wertebegriff ist die Entstehung individueller Werthaltungen bisher nur selten Gegenstand einer systematischen Diskussion gewesen (Schubarth 2016, S. 25) und auch empirisch bisher wenig beleuchtet worden. Zwei wichtige Ausnahmen sind hier jedoch zu nennen: entwicklungspsychologische und pädagogische Ansätze. In entwicklungspsychologischen Ansätzen - die stark von Piaget (1932) und Kohlberg (1984) geprägt wurden - wird argumentiert, dass sich die moralische Entwicklung einer Person über bestimmte hierarchisch Stufen vollzieht, deren Beginn bei der Perspektivenübernahme von primären Bezugspersonen verortet wird und sich im Laufe des Jugendalters auf größere Gruppen bis hin zu universalistischen Prinzipien ausbreitet. Nunner-Winkler und Kolleginnen beschreiben dieses „,biographische Erfahrungslernen“ folgendermaßen: ,[Es] ist der entscheidende Lernmechanismus. Eine besondere Bedeutung hat dabei zunächst die Identifizierung mit elterlichen Werthaltungen. Später kommen die Auseinandersetzungen mit Gleichaltrigen und mit Normierungen im weiteren sozialen Umfeld hinzu. Durch eigene Stellungnahme bestimmt dann der Heranwachsende seine Bindung an Moral (mit)“ (Nunner-Winkler et al. 2006, S. 43). ${ }^{1}$ Die zweite Ausnahme bilden pädagogische Überlegungen zur Gestaltung von Wertevermittlung und Wertebildung. Wertebildung wird dort als Prozess verstanden, ,in dem Menschen im Laufe ihrer Persönlichkeitsentwicklung Werte bzw. Werthaltungen entwickeln und Wertekompetenz [...] erwerben. Dieser Prozess vollzieht sich in der Auseinandersetzung des Individuums mit seiner sozialen Umwelt, vor allem durch das Erleben und Reflektieren von Werten“ (ebd.). Die Entstehung individueller Werthaltungen wird pädagogisch also auch in Schulen und anderen Bildungseinrichtungen verortet, und zwar als Wechselspiel von Vermittlung und Internalisierung von Werten. Auch empirische Untersuchungen zur Wertebildung befassen sich hauptsächlich mit der pädagogischen Vermittlung von Werten in Kindergärten und Schulen (Bertelsmann Stiftung 2016; Schubarth 2010). ${ }^{2}$ Meist steht die Prägung durch Lehrpersonen im Vordergrund solcher Untersuchungen (Standop 2005). Nach Lussi und Huber (2015) kommt aber generell schulischen Erfahrungen eine große Bedeutung zu, denn auch MitschülerInnen können Werthaltungen positiv oder auch negativ beeinflussen.

Etwas breiter sind die Erklärungskonzepte zur Entstehung individueller Werthaltungen in der Soziologie angelegt, die im Zusammenhang mit Bildung, Vermittlung und Internalisierung von Werten hauptsächlich mit Sozialisationsansätzen arbeiten. Dabei geht es um die vielfältigen Formen der gesellschaftlichen Einflussnahme auf individuelle Entwicklung; angenommen wird, dass im Sozialisationsprozess Werte erlernt und als Teil der Identität angelegt werden. Dieser Prozess läuft durchaus differenziert ab. Scherr (2013) und Castels et al. (2002, S. 114-115) argumentieren etwa, dass es in pluralen Gesellschaften nicht einen Bezugspunkt für Werte gibt,

\footnotetext{
1 Kohlbergs methodisches Vorgehen verweist außerdem auch auf die von uns in den Fokus gestellte Bedeutung von Konflikten zur Wertebildung. Kohlberg legte zur Befragung moralische Dilemmata vor und ordnete die Entscheidungen anhand ihrer Begründungslogik gewissen Entwicklungsstufen zu.

2 Einige AutorInnen argumentieren, dass schulische Erziehung und besonders intendierte Vermittlung nur einen Teil jenes Erfahrungsmaterials ausmachen, das im Sozialisationsprozess wirksam wird. Danach würde die Selbstsozialisation über den Kontakt mit Gleichaltrigen ebenso in den komplexen Aneignungsprozess von individuellen Werthaltungen hineinwirken wie Erfahrungen in anderen Lebensbereichen (Freizeit, Familie) (Scherr 2013, S. 276).
} 
sondern unterschiedliche gesellschaftliche Teilbereiche unterschiedliche Ansprüche an die Ausprägung individueller Werthaltungen stellen. Wie individuelle Werthaltungen während der primären und sekundären Sozialisation, aber besonders auch im späteren Lebensverlauf entstehen können, beschreibt der Soziologe und Sozialphilosoph Hans Joas ausführlich in seinem Buch ,Die Entstehung der Werte“. Joas Grundgedanke ist, dass Werte von Menschen weder gewählt, noch (absichtlich) in ihnen erzeugt werden könnten, stattdessen seien sie stark mit subjektiver Erfahrung verknüpft (Joas 1999). Werte würden daher zum einen durch das passive Moment des „Ergriffenseins“ entstehen, also durch Situationen, die uns in besonderer Art und Weise beeindrucken oder emotional berühren und daher an Wert gewinnen. Zum anderen würden Menschen Werte als eine Freiheit empfinden, sich selbst und der eigenen Identität ganz entsprechen zu dürfen (Joas 2006, S. 2). Für die Entstehung individueller Werthaltungen sind damit, wenn man Joas folgt, zwei Elemente zentral: Einerseits könnten Werte nur durch Selbstbildung, also in einem reflexiven Verhältnis zu sich selbst, entstehen. Im Laufe der menschlichen Entwicklung würden wir lernen, emotionale Beziehungen zu Menschen von unserer Einstellung zu ihren Werten zu trennen. Zweitens machen Menschen kollektive Erfahrungen, die durch ein ,über-sich-selbst-hinausgerissen-Werden“ (ebd., S. 7) charakterisiert sind und dadurch die Bildung von Werten entscheidend beeinflussen können. Die Entstehung individueller Werthaltungen werde daher auch durch „kollektive Ereignisse“ wie beispielsweise den Fall des Eisernen Vorhangs 1989 oder die Flüchtlingskrise von 2015/16 beeinflusst. Das von Joas beschriebene Konzept der Entstehung von Wertbindungen durch diese zwei Prozesse ist für die theoretischen Überlegungen des vorliegenden Beitrags zentral. Stein (2012) weist anknüpfend darauf hin, dass die Herausbildung von Werthaltungen ständigen Veränderungen unterlegen sein kann und außerdem von „biografischen Lebensereignissen“ (Stein 2013, S. 20) beeinflusst wird. Werte würden ,sehr stark in informellen Kontexten und in indirekten, nicht intentionalen Prozessen erworben" werden.

\section{Methoden und Daten}

An die zuvor vorgestellten Forschungsarbeiten knüpfen wir an und fragen mit unserem Beitrag danach, wie sich Werte durch Erfahrungen in verschiedenen Lebensbereichen (neben Familie zum Beispiel durch Arbeit oder sozialpolitisches Engagement) entfalten und ob es Unterschiede in der Bedeutung dieser Bereiche für die Wertebildung verschiedener sozialer Gruppen gibt. Dazu arbeiten wir in der vorliegenden Studie mit einem Mixed-Methods-Ansatz im „Explorativen Design“ (Creswell und Plano Cark 2011, S. 75-76). Auf eine explorative qualitative Phase, in der offen nach wichtigen Konzepten und deren Bedeutung im Feld gesucht wird, folgt hier eine quantitative Phase, in der verallgemeinernde Aussagen über die Entstehung individueller Werthaltungen in Österreich getroffen werden. 


\subsection{Fokusgruppen}

In der ersten Phase (A) wurden acht Fokusgruppendiskussionen mit Menschen in Österreich durchgeführt. Die leitende Forschungsfrage war, was ÖsterreicherInnen für die Bildung ihrer eigenen Werte als entscheidend wahrnehmen. Ein besonderer Fokus lag dabei auf den Orten und Prozessen, in die diese prägenden Erlebnisse eingebettet sind. Zwischen November 2015 und April 2016 wurden dazu 35 Personen in Kleingruppen befragt. Wir haben uns dabei an einer Maximum-Variation-SamplingStrategie orientiert (Suri 2011) und versucht, möglichst unterschiedliche Gruppen in Österreich zu erreichen, ${ }^{3}$ um Wertevorstellungen in der Gesellschaft kontrastreich abzubilden. Die Rekrutierung der StudienteilnehmerInnen erfolgte über Anzeigen in verschiedenen Zeitungen (u.a. Die Krone, Das Biber, Bezirksblatt Eisenstadt), Webseiten und Flugzetteln in Supermärkten. Das daraus resultierende Sample enthielt 22 Frauen und 13 Männer und es sind alle Altersgruppen zwischen 17 und 67 Jahren vertreten. Die meisten TeilnehmerInnen waren erwerbstätig, sieben Personen waren im Ruhestand. 15 Personen wiesen einen Migrationshintergrund auf (z.B. Türkei, Deutschland, Schweden, Rumänien, Ex-Jugoslawien, Iran), wobei die meisten der sogenannten ersten Generation zuzurechnen sind. In unserer Untersuchungsgruppe finden sich einige Personen mit geringen Qualifikationen, mittlere und hohe Bildungsabschlüsse sind etwas stärker vertreten. ${ }^{4}$ In den Fokusgruppeninterviews wurden verschiedene Themen aufgegriffen, u. a. die Deutung des Wertebegriffs durch die Befragten; Aussagen über eigene Werthaltungen und Schilderungen zur Vermittlung oder Prägung der eigenen Werte. Für den vorliegenden Beitrag haben wir uns auf die Erfahrungen konzentriert, die für die Entstehung der eigenen Werte als besonders prägend empfunden wurden. Bei der Analyse des qualitativen Textmaterials wurde mit der Dokumentarischen Methode nach Bohnsack gearbeitet (Bohnsack et al. 2013), wobei besonders die formulierende Interpretation, also die manifeste Beschreibung des Datenmaterials, Basis der folgenden Ausführungen ist. Dabei konnten sowohl vertiefende Analysen einzelner Fälle als auch fallübergreifende Vergleichsanalysen vorgenommen werden.

\subsection{Standardisierter Survey}

\subsubsection{Datensatz}

Um die Ergebnisse aus den Fokusgruppendiskussionen quantitativ zu kontextualisieren und zu vertiefen, wurde in einem zweiten Schritt (B) ein Fragebogen zur Entstehung individueller Werthaltungen entwickelt. Der Fragebogen enthält zum Teil standardisierte Items der internationalen Umfrageforschung (basierend auf Studien

\footnotetext{
3 Eine besondere Herausforderung bestand darin, die Gruppen gleichzeitig nach innen hin möglichst homogen zu gestalten, da gemeinsame Erfahrungshorizonte den Austausch in Fokusgruppen erleichtern (Bryman und Bell 2015, S. 520).

4 Das Sample enthält Menschen mit unterschiedlichsten biografischen Erfahrungen und aus verschiedenen sozialen Milieus. Limitationen ergeben sich durch den Umstand, dass Menschen mit geringer Bildung und Männer weniger stark vertreten sind als geplant.
} 
wie dem ALLBUS 2014, vgl. GESIS 2014). Ein zentraler Schritt der quantitativen Teilstudie war jedoch die Entwicklung neuer Skalen zur Entstehung individueller Werthaltungen, basierend auf den Ergebnissen der Fokusgruppendiskussionen. Diese Skalen beziehen sich inhaltlich unter anderem auf prägende Erlebnisse im Leben, die von den Befragten als wertebildend angegeben wurden. Im Rahmen des Projektes wurden die Skalen in einer onlinebasierten Umfrage eingesetzt $(n=1500)$ und damit erstmalig für Österreich getestet. Die Stichprobe ist repräsentativ für die österreichische Bevölkerung zwischen 15 und 69 Jahren in Bezug auf Alter, Geschlecht, Bildungsgrad und Bundesland und deckt sich mit der Verteilung zentraler sozialstruktureller Merkmale im gewichteten Mikrozensus 2015. Lediglich MigrantInnen der ersten Generation sind in den verwendeten Daten leicht unterrepräsentiert.

\subsubsection{Abhängige Variable}

Die für die Analysen des vorliegenden Beitrags herangezogene Variable - Bewertung der Prägung verschiedener Lebensbereiche auf die eigenen Werthaltungen - gehört zu diesen neu entwickelten Skalen. Sie gliedert sich in zwei Teile: Zunächst werden die Befragten um eine Einschätzung der Prägekraft von elf Lebensbereichen auf ihre individuellen Werthaltungen gebeten (vierteilige Skala: 1= gar nicht geprägt, $2=$ schwach geprägt, $3=$ eher stark geprägt, $4=$ sehr stark geprägt), die in den Fokusgruppen besonders intensiv diskutiert wurden. Wie im Artikel noch ausführlich dargelegt wird, berichteten FokusgruppenteilnehmerInnen, dass sowohl positive als auch negative Ereignisse prägend für ihre individuellen Werthaltungen waren. Die im zweiten Teil der Skala vorgenommene Einschätzung der Wertprägung ${ }^{5}$ rangierte entsprechend zwischen positiver und negativer Prägung $(1=\operatorname{sehr}$ positiv, $2=$ eher positiv, $3=$ teils/teils, $4=$ eher negativ, $5=$ sehr negativ). Als abhängige Variable für die Berechnungen logistischer Regressionen wurde diese Einschätzung der Werteprägung in eine binäre Variable umgewandelt (,negative Prägung durch wichtige Lebensbereiche“: $1=$ teils/teils bis sehr negativ; $0=$ alle anderen Bewertungen).

\subsubsection{UnabhängigeVariablen}

Für die unabhängigen Variablen wurde auf etablierte Messmodelle zurückgegriffen. In die logistische Regressionsanalyse fließen danach ein: Geschlecht ( 1 = männlich, 2 = weiblich), Alter (1 = 15-29 Jahre, 2 = 30-39 Jahre, 3 = 40-49 Jahre, $4=50-59$ Jahre, 5=60-69 Jahre), Bildung ( $1=$ kein Abschluss oder Pflichtschulabschluss, 2 =Lehrausbildung oder berufsbildende mittlere Schule, $3=$ höhere allgemein oder berufsbildende Schule oder Kolleg ohne akad. Abschluss, 4 = Universitätsoder Fachhochschulabschluss), Migrationshintergrund $(0=$ kein Migrationshintergrund, $1=$ eigene Staatsbürgerschaft oder Geburtsland im Ausland, oder jenes mind. eines Elternteils) und der Grad der Religiosität ( $1=$ nicht religiös, $2=$ eher religiös, $3=$ stark religiös). Als weitere Kontrollvariablen dienen: die Lebensregion bzw. der Sozialraum (1= unter 30.000 Einwohner, 2=zwischen 30.000 und 300.000 Einwoh-

\footnotetext{
5 Der Fragetext lautete: „Es gibt Erfahrungen, die uns im Leben stark prägen. Bitte geben Sie an, inwiefern Sie von den genannten Erfahrungen geprägt wurden“".
} 
ner, $3=$ über 300.000 Einwohner) und die politische Positionierung ( $1=$ eher links, $2=$ Mitte, $3=$ eher rechts).

\subsubsection{Methode}

Zunächst wird durch deskriptive Analysen der Frage nachgegangen, wie sich die Lebensbereiche in ihrer Prägkraft aus Sicht der ÖsterreicherInnen prinzipiell unterscheiden. In einem zweiten Schritt wurde mittels binär logistischer Regressionsmodelle die Verteilung negativer Prägungserfahrungen innerhalb der österreichischen Bevölkerung für drei zentrale Lebensbereiche untersucht: Elternhaus, Partnerschaft und biografische Brüche. Diese Lebensbereiche wurden exemplarisch auf Basis der Fokusgruppen ausgewählt.

\section{Empirische Befunde zur Entstehung individueller Werthaltungen in Österreich}

\subsection{Orte der Werteprägung}

Bisherige Studien fokussieren darauf, dass individuelle Werthaltungen hauptsächlich während der Sozialisation und durch Vermittlungsprozesse, z. B. in Schulen, entstehen. Wir haben dies als Ausgangspunkt genommen und in den Fokusgruppendiskussionen offen die Eingangsfrage gestellt, welche Erfahrungen, Ereignisse und Lebensbereiche von ÖsterreicherInnen als prägend für ihre Werte wahrgenommen werden. Die Fülle der folgenden Erzählungen wurde im Zuge der Auswertungen und Textinterpretation in mehrere zentrale Lebensbereiche zusammengefasst. Im Zuge der formulierenden Interpretation wurde zunächst die thematische Struktur der Gesprächsprotokolle entschlüsselt. Anschließend wurden in einer vergleichenden Analyse die Textstellen zu einem Thema gesammelt und Gemeinsamkeiten und Unterschiede zwischen den Gruppen herausgearbeitet. Dabei wurde die Abgrenzung der Themen voneinander verfeinert. Das Resultat sind die Identifizierung von elf Lebensbereichen, in deren Kontext die Befragten von werteprägenden Erfahrungen berichten:

- Das eigene Elternhaus

- Partnerschaft

- Freunde

- Schulbildung

- Berufsausbildung (inkl. Universitätsausbildung)

- Arbeit

- Migrations- und Auslandserfahrungen

- Biografische Brüche (z. B. Schicksalsschläge, Unfälle, Todesfälle)

- Engagement in Freizeitorganisationen (z. B. Sportverein)

- Engagement in sozialen oder politischen Organisationen (z. B. Unterstützung für geflüchtete Menschen)

- Engagement in religiösen Organisationen (z. B. Kirchengemeinde) 
Die Übersicht macht klar, dass die Entstehung individueller Werthaltungen, auch von den Befragten selbst, als sehr weitreichender und komplexer Prozess wahrgenommen wird, der in viele Bereiche des Lebens hineinreicht. Um den Facettenreichtum dieser Wertebildungsprozesse zu veranschaulichen, werden im Folgenden zwei exemplarische Lebensbereiche dargestellt: die Familie (Elternhaus und Partnerschaft) und biografische Brüche. Diese beiden Bereiche wurden aus mehreren Gründen ausgewählt: Zunächst waren die Prägung durch Familie und biografische Brüche in allen Fokusgruppen vertreten. Gleichzeitig wurde Wertebildung durch diese Erfahrungen besonders emotional und facettenreich diskutiert, ein Indiz für ihre hohe Bedeutsamkeit aus Sicht der Befragten (Bohnsack und Przyborski 2006; Joas 1999). Zuletzt veranschaulichen diese beiden Bereiche auch den Beitrag, den wir durch die explorative Mixed-Methods-Ausrichtung des Projektes für die Werteforschung leisten können: Die Prägung durch Familie steht dabei exemplarisch für ähnliche Bereiche wie Prägung durch Freunde, Schule und Religion, welche bereits in der Literatur aufgegriffen werden. Trotzdem versucht der vorliegende Beitrag aufzuzeigen, dass sich durch die Offenheit und Interaktivität der Fokusgruppendiskussionen neue Aspekte der Entstehung individueller Werthaltungen auch in diesen Bereichen finden lassen. Biografische Brüche hingegen wurden in empirischen Studien zur Entstehung individueller Werthaltungen bisher kaum erwähnt. Hier kann mithilfe der offenen Herangehensweise der qualitativen Einstiegsphase ein bisher weitgehend unbekannter Prägungsprozess diskutiert werden.

\subsection{Qualitative Ergebnisse zur Entstehung individueller Werthaltungen}

\subsubsection{Familie: Elternhaus und Partnerschaft}

Die Forschungsliteratur thematisiert immer wieder, wie bedeutsam die Familie im Zusammenhang mit der Entstehung individueller Werthaltungen ist, wobei besonders dem Elternhaus eine entscheidende Rolle zugeschrieben wird (u. a. Fend et al. 2009; Grusec und Kczynski 1997; Kohn et al. 1986). Welche familienspezifischen Bedingungen Einfluss nehmen und wie die Entstehung von Werten im Kontext von Familie tatsächlich konkret vonstattengehen kann, ist dagegen bisher selten aus soziologischer Perspektive thematisiert worden (vgl. Stein 2013). Mit den vorliegenden Befunden können wir dieses Desiderat aufgreifen und eine Reihe an Facetten und exemplarischen Abläufen des Wertebildungsprozesses im Kontext von Familie beleuchten. Wie sich Werte im Kontext der Familie (v.a. im Elternhaus, aber auch in Partnerschaften) bilden (können), wird mit unseren qualitativen Daten aus Sicht von Erwachsenen retrospektiv beschrieben. Aus diesem Blickwinkel ist die Entstehung individueller Werthaltungen ein facettenreicher Prozess, der keinem linearen Ablauf folgt, sondern sich vor allem auch durch Konflikthaftigkeit, Abgrenzung gegenüber Werthaltungen anderer Personen und/oder Familienmitgliedern vollzieht und teils mit ambivalenten Bewertungen verknüpft ist, was auch später in den Analysen des quantitativen Materials sichtbar wird.

Zunächst lässt sich in Anlehnung an Stein (2013) feststellen, dass Werte im Kontext von Familie eher in nicht intendierten Situationen gebildet werden. So fällt es den Befragten in den Fokusgruppendiskussionen anfänglich auch schwer, konkrete 
Erlebnisse zu berichten und ihre Schilderungen zur Entstehung individueller Werthaltungen wiesen zunächst eher allgemeinen Charakter auf. Viele der Befragten beriefen sich in ihren Schilderungen auf gemeinsame Erfahrungen und Wissensbestände hinsichtlich der Prägekraft der Familie, auch wenn sie zunächst keine konkreten Einzelheiten schilderten. Unsere Befunde legen deshalb nahe, dass die Entstehung individueller Werthaltungen im Kontext von Familie zunächst als ein eher unbewusst wirkender Prozess wahrgenommen wird, der erst in der Rückschau genauer benannt werden kann. Während also der Verlauf dieser Prägungserfahrung meist nicht explizit artikuliert werden konnte, wurde stattdessen in den Fokusgruppen auf das „Ergebnis“ dieses Vorganges, nämlich die implizite Weitergabe von „Grundwerten“, verwiesen. Dieses Ergebnis schließt aus unserer Sicht an klassisch soziologische Wertekonzepte an, in denen argumentiert wird, dass Werte abstrakte oder generalisierte Inhalte sind, die Gemeinsamkeit unterstellen und als ,höhere Prinzipien“"gelten (u.a. Spates 1983; Thome 2003).

In den Fokusgruppen galten besonders die Eltern als Vorbilder für die eigenen Werte, die ein „Grundgerüst“ und damit eine Basis für das weitere Leben vermitteln würden. Als Beispiele wurden hier Unterstützung, Rückhalt und Vertrauen als grundlegende Werte genannt, die implizit über Praxis vermittelt worden seien und eine Basis für den eigenen Lebensweg bilden würden. So erzählt eine Teilnehmerin, Anfang dreißig: „Meine Eltern haben mich zu dem gemacht, was ich bin, also über die Erziehung [...] [sie] waren wahrscheinlich am prägendsten, weil sie dann geformt haben, wohin ich will " (FG 3). ${ }^{6}$ Diese Befunde zur Relevanz der Eltern für die Entstehung individueller Werthaltungen schließt u.a. an Erkenntnisse von Welzel an. Im Anschluss an Allport und Hurrelmann geht er davon aus, dass während der Sozialisation bestimmte Wertprofile ausgebildet werden, die die Identität eines Individuums nachhaltig beeinflussen (Welzel 2009, S. 109). Dieser Prozess ist langfristig angelegt und auf die Zukunft ausgerichtet, wie ein Teilnehmer berichtet: „das gibt einen Weg vor, wie man sein restliches Leben bestreitet, also ist das auch [...] wertegebend, ja“ (FG 3). Ein wichtiger Aspekt der Wertebildung durch elterliche Einflüsse ist die Annahme, dass eine starke Prägung durch sie immer dann als entscheidend angesehen wurde, wenn die Eltern ihre Werte ,,authentisch“ vorlebten (Stein 2013). Eine Teilnehmerin, die in einem religiös-praktizierenden Elternhaus aufgewachsen ist, berichtet: „, ich habe gespürt, dass diese Werte tragen, die sie mir vermitteln, das war nicht irgendwas [...] meine Eltern haben das authentisch gelebt" (FG 8). Diese Authentizität begründet die Befragte damit, dass ihre Eltern durch die Herstellung kohärenter Bezüge zwischen Denken und Handeln als Vorbilder für sie fungierten. Das von der Befragten thematisierte Erleben der Eltern, die offensichtlich authentisch versuchten, gedankliche Konstrukte praktisch umzusetzen, dürfte jener wertebildenden Erfahrung entsprechen, die Joas als „Ergriffensein“ bezeichnet hat: „das Gefühl, ganz besonders mit uns identisch zu sein“ (Joas 2006, S. 2).

Die Analysen unserer Fokusgruppen legen nahe, dass die Entstehung individueller Werthaltungen im Kontext der Familie zu großen Teilen als nicht-intendiert und vorwiegend unbewusst abläuft. Einige Befragte ergänzen für die Lebensabschnitte

\footnotetext{
${ }^{6} \mathrm{FG}=$ Fokusgruppe.
} 
der Pubertät und des jungen Erwachsenenalters, dass die Werte der Eltern mit dem Heranwachsen zunehmend, teilweise jedoch auch nur vorübergehend, als Kontrastfolie zu eigenen Werten dienten und in manchen Fällen auch Ausgangspunkt für Konflikte bildeten. Einige äußerten, gegen die Werte der Eltern rebelliert zu haben bzw. diese abzulehnen, und dass genau dieser Prozess zur Entwicklung einer eigenen Persönlichkeit mit eigenen Werthaltungen geführt habe (vgl. Joas 1999, 2006). Eine Teilnehmerin berichtet, sich im Erwachsenenalter gegen die sehr strengen Erziehungsmethoden der Eltern gewendet zu haben: „Ich habe mich [...] von diesen Werten distanziert, die ich in der Kindheit zu spüren bekommen hab, [...] die wollte ich nicht annehmen “, berichtet sie und erzählt weiter: „, ich hab das eher so erlebt, die Eltern prägen mich und ich hab jahrelang damit zu tun, das wieder loszuwerden, was sie in mich hineingeprägt haben, bis ich ein freier Mensch sein kann" (FG 8). Die Befragte rückte also von den elterlichen Werten ab, indem sie sich, wie sie im Gespräch beschreibt, Vorbildern zuwandte, die ihrer Meinung nach und im Gegensatz zu den Eltern wichtige Werte wie „Freiheit“ und „Liebe“ vertraten. In ihrem Fall bestand die Konsequenz ihrer Distanzierung zu den elterlichen Werten darin, sich einer religiösen Gemeinschaft anzuschließen, da sie hier die Vorbilder und Werte fand, nach denen sie leben wollte. Dieser Abwendungs-Prozess entspricht einem typischen Ablauf der Entstehung individueller Werthaltungen in unserer Untersuchungsgruppe.

Neben den Eltern wurden in den Fokusgruppen vereinzelt auch andere Familienmitglieder erwähnt, wie Großeltern, Geschwister, Kinder oder entferntere Verwandte. Eine weitere wichtige Rolle nahmen in vielen Fokusgruppen die Partnerschaften ein, die die Befragten in ihren Werten ,bereichern“ und „verändern“ (FG 1). Als besonders prägend wurde dabei erneut häufig der Umgang mit Konflikten oder dem Scheitern einer Beziehung genannt. In einer Diskussionsrunde berichteten etwa gleich mehrere Personen von der Erfahrung, sich nach langen Jahren von ihren Partnern scheiden zu lassen: „Der Entschluss mich scheiden zu lassen nach 11 Jahren, die in den letzten Jahren ziemlich wüst waren [...], das war schon sehr einschneidend für mich. [...] Mir war dann die Unabhängigkeit und Fähigkeit zur Selbstversorgung äußerst wichtig, und das ist es auch bis heute" (FG 4). Zudem reflektierten viele ältere GruppenteilnehmerInnen einen Wertewandel in Partnerschaften, der vor allem an der stärkeren rechtlichen Unabhängigkeit der Frau in der Beziehung (Unterhalt, Vormundschaft, Erwerbsarbeit) und an veränderten Einstellungen zu Sexualität festgemacht wurde.

\subsubsection{Biografische Brüche}

Dass Biografien von Diskontinuitäten und unterschiedlichen Übergangsphasen (beispielsweise von der Schule in die Berufstätigkeit) geprägt sind, ist u. a. aufgrund der Erkenntnisse der Lebenslauf- und Biografieforschung bekannt (u. a. Hoerning 1987; Kohli 1988). „Biografische Wendepunkte“ sind aber beispielsweise auch im Zusammenhang mit der Arbeitslosigkeitsforschung oder der Kriminalsoziologie (u.a. Vogel 2008) untersucht worden. Im Kontext der Entstehung individueller Werthaltungen wird dieses Thema jedoch bislang selten diskutiert. Ausnahmen bilden hier die bereits in Abschn. 2 erwähnten Arbeiten von Stein sowie Nummer-Winkler et al., 
die in diesem Kontext „biografische Lebensereignisse“ wie beispielsweise die „Begegnungen mit neuen Menschen“ als werteprägend hervorheben (Nunner-Winkler et al. 2006, S. 43; Stein 2013, S. 11, 20).

Biografisch prägende Erfahrungen können für die Entstehung individueller Werthaltungen nach unseren Befunden sehr relevant sein, wobei die TeilnehmerInnen der Fokusgruppendiskussionen häufig von ähnlichen Erfahrungen und Erlebnissen berichteten. Biografische Brüche werden in unserer Studie als plötzliche, unerwartete oder unbekannte Erfahrungen beschrieben, die zu einer grundlegenden Neuorientierung oder einer Neubewertung des eigenen Lebens führten. Als Beispiele können hier Unfälle, der Tod eines Angehörigen, aber auch Scheidungen, Arbeitslosigkeit oder sogar längere Auslandsaufenthalte und das Erleben neuer Lebenskontexte genannt werden. Obwohl sich die konkreten Lebensereignisse der Befragten in Teilen voneinander unterscheiden, lassen sich einige allgemeine Facetten für die Entstehung individueller Werthaltungen im Kontext biografischer Brüche aufzeigen. Gemeinsam ist diesen Aspekten, dass diese Erlebnisse meist nicht nur die Person selbst, sondern auch ihr unmittelbares soziales Umfeld einschließen (Familie, FreundInnen, KollegInnen). Zudem zeichnen sich biografische Brüche dadurch aus, dass sie mit dem Gefühl eines erzwungenen Verlusts eigener Werte einhergehen (beispielsweise Verlust der Selbstbestimmung durch Krankheit), und diese Werte dadurch in ihrer Geltung bestätigen und verfestigen. ${ }^{7}$ Diese und ähnliche Befunde aus unseren Fokusgruppen korrespondieren mit Joas Wertekonzept, nach dem gerade solche Situationen zur Bildung von Werten führen können, die uns in einer besonderen (positiven oder negativen) Weise beeindrucken und ,ergreifen“ (Joas 1999, S. 206-207, 2006, S. 2). Typisch ist auch diese rückblickende Äußerung einer jungen Teilnehmerin, in der bestimmte Erlebnisse und Erfahrungen als wesentliche Markierungen geschildert werden: ,wenn dir etwas sehr Schreckliches [...] oder was Positives passiert, das [hat] einen Einfluss auf die Werte des Menschen und auf die Sichtweise, wie er durch das Leben geht" (FG 6).

In jedem Fall bedeuteten biografische Brüche immer „Umstrukturierungen, Veränderungen, Neujustierung“ formuliert Hoerning (1987, S. 237). Das Material unserer Fokusgruppendiskussionen legt nahe, dass sowohl positiv als auch negativ empfundene biografische Brüche als prägend für die Entstehung individueller Werthaltungen wirken können.

$\mathrm{Zu}$ den werteprägenden biografischen Ereignissen gehört auch das Erleben neuer Kontexte. Dabei wurden beispielsweise Lebensstationen oder Reisen im Ausland von einigen Befragten als werteprägend beschrieben, da man durch das Kennenlernen neuer Kulturen und Werte zur Selbstreflexion eigener Positionen angehalten sei. Ein junger Teilnehmer erzählt: „Wenn man wirklich in einen neuen Kontext kommt, sich neu orientieren muss [...] da [finden] die größten Veränderungen statt, auch mit einem selber, weil man vieles, was man bisher gemacht hat, hinterfragt“. Dieses Erleben neuer Kontexte führt zur Hinterfragung von Handlungsmustern, die bisher vorausgesetzt wurden. Die Nicht-Bestätigung von Bekanntem führt in diesem Fall

\footnotetext{
7 Eine Teilnehmerin beschreibt dies exemplarisch so: „Es wird einem [...] erst vielleicht eigentlich später klar, [dass] so Verlustsachen, so Gesundheitssachen [...] einen eigentlich dann doch mehr prägen, als man das zu dem Zeitpunkt wahrnimmt" (FG 3).
} 
zu einer verstärkten Reflexion eigener Denk- und Verhaltensweisen, die auch in die (Neu-)Bildung von Werten münden kann. Obwohl verschiedene AutorInnen auf die zeitliche Stabilität von Werten hinweisen (u.a. Thome 2003; Welzel 2009), zeigen unsere Befunde, dass sich Werthaltungen durch Auseinandersetzung und Konfrontation durchaus verändern können, auch wenn der Prozess selbst auf längere Zeiträume angelegt ist. Das Erleben neuer Kontexte, besonders im Kontext von längeren Auslandsaufenthalten, erforderte immer ,neue Ordnungsmuster aufzubauen, die die eigene Identität herausfordern“ (Bachleitner und Aschauer 2015, S. 339). Dabei waren Offenheit und Eigenständigkeit die Werte, die in diesem Zusammenhang besonders oft genannt wurden. „Alleine was Selbständigkeit anbelangt [...]“, erzählt eine Teilnehmerin, ,, ich glaube, das andere Umfeld prägt da schon sehr stark“ (FG 1). Auch eine andere Befragte, die aufgrund beruflicher Umstände in anderen Ländern lebte und dies als stark prägend empfand, berichtete in der Fokusgruppe von der Bedeutung dieser Auslandsaufenthalte: „Wenn man den Wohnort ändert oder raus aus der eigenen Komfortzone geht und irgendetwas Neues erlebt, das ist schon etwas, woran man sich dann in der Retrospektive erinnert und dem wahrscheinlich auch beimisst, dass man sich sehr verändert hat" (FG 3). Erfahrungen dieser Art werden als wichtige Stationen im Leben wahrgenommen, die zu Reflexion und persönlicher Weiterentwicklung anregen und damit Auswirkungen auf das eigene Werteprofil haben (vgl. Welzel 2009).

$\mathrm{Zu}$ den biografischen Brüchen als wertebildende Momente im Leben zählen aber auch tiefgreifende Zäsuren wie (chronische) Krankheiten, Unfälle oder das Erfahren körperliche Gewalt. ${ }^{8}$ In diesem Zusammenhang wurde von den Befragten besonders der Verlust von Selbstständigkeit thematisiert, der im weiteren Lebensverlauf dazu führte, Selbstbestimmung und Autonomie neue Bedeutung beizumessen. Ein junger Befragter, der während seiner Kindheit körperliche Gewalt erfahren hatte, berichtete dazu: „Das macht natürlich viel mit einem [...] im Nachhinein betrachtet absolut [etwas] Positives, weil man unglaublich viel aus der Situation lernt, auch fürs spätere Leben, wie man auch auftreten muss gegenüber anderen “ (FG 1). Biografische Brüche führten in einigen Fällen jedoch nicht nur zu einer persönlichen Veränderung, sondern auch zu einer verstärkten Neubewertung und Wertschätzung des eigenen Lebens. So beschrieb ein Befragter, der mehrere Wochen schwer erkrankt war und dies als besonders drastischen Einschnitt in sein Leben schilderte: „Ja, bei mir waren einige Sachen, wo sich eine Türe geschlossen, aber eine andere geöffnet hat“, berichtete er und erzählte weiter: „speziell auch vor zwei Jahren, wo ich im Koma lag, [...] aber mich wieder gut gefangen und natürlich dann viel bewusster nachher alles empfunden habe bzw. noch immer empfinde" (FG 2). Der Befragte reflektiert durch den erlebten Einschnitt seine bisherigen Lebenserfahrungen neu. Seine Erkrankung war für ihn außerdem die Bedingung dafür, dass sich neue Möglichkeiten der Lebensführung überhaupt eröffnen konnten. Dieses Beispiel zeigt, dass biografische Brüche in der Rückschau von den Befragten auch positiv bewertet und (um-)gedeutet werden, wenn sie in den eigenen Erfahrungshorizont

\footnotetext{
${ }^{8}$ Hervorzuheben ist, dass bei den Befragten unserer Studie für die Entstehung individueller Werthaltungen Konflikte oder Handlungsschwierigkeiten eine größere Rolle zu spielen scheinen als positiv erlebte Erfahrungen.
} 
eingebettet werden. Gleichzeitig sind „Lebensereignisse, die Verluste darstellen [...] für das Individuum eine Herausforderung, die [es] dazu veranlassen, ,neue 'Wege zu betreten“ (Hoerning 1987, S. 235). Andere Befragte, die ähnlich prägende Erfahrungen gemacht haben, berichteten davon, einen bewussteren und intensiveren Zugang zu ihrem Leben zu empfinden, aktiver zu werden und Pläne für die Zukunft nicht mehr länger aufzuschieben. „Das war also der Moment“, berichtete eine Pensionistin „wo ich beschlossen hab, ich schiebe überhaupt nichts mehr auf die Zukunft“ (FG 4). Darüber hinaus berichteten andere Personen in den Fokusgruppen sogar, dass einschneidende biografische Brüche zur Wahl eines bestimmten Berufes (z. B. Medizin) beigetragen hätten. Diese Befunde deuten auf jene theoretische Erkenntnisse hin, die davon ausgehen, dass Werte einen handlungsmotivierenden Charakter haben können, besonders dann, wenn sie mit Emotionen verknüpft sind (Schwartz 1994, S. 21).

Der konkrete Prozess der Entstehung individueller Werthaltungen lässt sich mit Blick auf die Befunde unserer Fokusgruppendiskussionen in folgender Weise zusammenfassen: Zunächst wurden bestimmte Werte, die von den Eltern vorgelebt wurden, von den Befragten in der Kindheit meist unbewusst übernommen. Durch das Heranwachsen kam es dann aber zu einem Reflexionsprozess, in dem diese Werte hinterfragt wurden. Hierzu zählt auch das Aufbegehren gegen oder Ablehnen von Werten der primären Bezugspersonen. In dieser Phase werden auch andere Beziehungen zu PartnerInnen oder FreundInnen für die persönliche Wertebildung sehr wichtig. Biografische Brüche und negative Erfahrungen innerhalb dieser Beziehungen werden von den Befragten als besonders prägend beschrieben, da sie Werte bewusst machen und auf die Probe stellen. Erst nach diesem Prozess, so berichteten einige Befragte, könnten sie angeben, eigene Werte und Einstellungen entwickelt zu haben. Ein Befragter fasst diesen Prozess eindrücklich zusammen: „Man [bekommt] natürlich von den Eltern mal so ein Grundgerüst an Werten und [richtet] sich erst mal nur nach dem, was einem vorgelebt wird. Und dann später in Konfrontation mit Freunden, mit anderen Leuten, da merkt man, was hat man dort für Werte? Was haben Freunde für Werte? Passt dich vielleicht da ein bisschen an, merkst dann, wo stimmt's nicht mehr zusammen mit dem, was man selber kennt-und baut sich dann halt langsam so sein eigenes Ding zusammen “ (FG 6).

\subsection{Quantitative Ergebnisse zur Entstehung individueller Werthaltungen}

\subsubsection{Bewertung der Entstehung individueller Werthaltungen}

Die bisher diskutierten Ergebnisse konnten zeigen, dass die Entstehung individueller Werthaltungen ein Prozess ist, der viele Lebensbereiche umfasst. Anhand unserer quantitativen Daten soll in einem weiteren Schritt vertiefend untersucht werden, wie stark die ÖsterreicherInnen die jeweiligen Lebensbereiche als prägend für ihre eigenen Werte empfinden. Zudem wurde in den Fokusgruppen auch sichtbar, dass individuelle Werthaltungen nicht nur in intendierten und positiven Vermittlungsprozessen entstehen, sondern dafür auch negativen Erfahrungen und Konflikten eine besondere Bedeutung zukommt. Deshalb wurde nicht nur die Stärke des Einflusses auf die eigenen Werthaltungen statistisch erfasst, sondern auch abgefragt, ob 
individuelle Werthaltungen in den verschiedenen Lebensbereichen eher positiv oder negativ geprägt wurden.

In Tab. 1 sind dazu die deskriptiven Ergebnisse im Überblick aufgeführt. Wie schon die qualitativen Ergebnisse zeigen, spielt die Sozialisation in der Familie für viele Menschen eine große Rolle für die Entstehung individueller Werthaltungen. Gleichzeitig zeigt sich auch, dass dieser wichtige Lebensbereich von vielen Personen durchaus ambivalent bewertet wird. Über $30 \%$ der ÖsterreicherInnen geben an, durch ihre Familie zumindest teilweise negativ geprägt worden zu sein. Der Umstand, dass die Entstehung individueller Werthaltungen in Familien auch als schmerzhafter Prozess erlebt werden kann, wird in der bisherigen Forschungsliteratur noch zu wenig reflektiert. Ein ebenfalls bisher wenig untersuchter Einflussfaktor auf die Entstehung individueller Werthaltungen ist die Partnerschaft. Wie Tab. 1 zeigt, wird diese von den Befragten als zweitwichtigster Ort der Wertebildung wahrgenommen. Erneut sind jedoch negative Erfahrungen für mehr als ein Drittel der in Österreich lebenden Personen (zumindest teilweise) vorherrschend.

Unsere Daten bestätigen auch die starke Prägungskraft von biografischen Brüchen, wie dies weiter oben bereits ausführlicher diskutiert wurde. Zwei Drittel der ÖsterreicherInnen geben an, durch solche Brüche in ihren Werten stark geprägt worden zu sein. Ein Großteil der Befragten empfindet diese Prozesse als negativ; viele

Tab. 1 Die Entstehung individueller Werthaltungen in verschiedenen Lebensbereichen: Stärke und Wahrnehmung der Prägung (in \%)

\begin{tabular}{|c|c|c|c|c|c|c|c|c|c|}
\hline & $\begin{array}{l}\text { Gar nicht } \\
\text { geprägt }\end{array}$ & $\begin{array}{l}\text { Schwach } \\
\text { geprägt }\end{array}$ & $\begin{array}{l}\text { Eher stark } \\
\text { geprägt }\end{array}$ & $\begin{array}{l}\text { Sehrstark } \\
\text { geprägt }\end{array}$ & $\begin{array}{l}\text { Sehr } \\
\text { po- } \\
\text { sitiv }\end{array}$ & $\begin{array}{l}\text { Eher } \\
\text { po- } \\
\text { sitiv }\end{array}$ & $\begin{array}{l}\text { Teils/ } \\
\text { teils }\end{array}$ & $\begin{array}{l}\text { Eher } \\
\text { ne- } \\
\text { gativ }\end{array}$ & $\begin{array}{l}\text { Sehr } \\
\text { ne- } \\
\text { gativ }\end{array}$ \\
\hline \multicolumn{10}{|l|}{ Familie } \\
\hline Elternhaus & 2 & 10 & 41 & 48 & 39 & 28 & 24 & 6 & 3 \\
\hline Partner & 3 & 14 & 39 & 43 & 33 & 31 & 24 & 9 & 3 \\
\hline Freunde & 2 & 20 & 50 & 28 & 25 & 42 & 30 & 4 & 1 \\
\hline \multicolumn{10}{|l|}{ Ausbildung } \\
\hline Schule & 4 & 32 & 48 & 17 & 10 & 35 & 41 & 12 & 2 \\
\hline $\begin{array}{l}\text { Berufsaus- } \\
\text { bildung }\end{array}$ & 5 & 28 & 47 & 21 & 17 & 41 & 35 & 5 & 1 \\
\hline Arbeitsleben & 3 & 20 & 46 & 31 & 18 & 39 & 34 & 7 & 2 \\
\hline $\begin{array}{l}\text { Biografische } \\
\text { Brüche }\end{array}$ & 7 & 26 & 31 & 36 & 6 & 13 & 43 & 23 & 15 \\
\hline $\begin{array}{l}\text { Migration/ } \\
\text { Auslands- } \\
\text { erfahrung }\end{array}$ & 16 & 36 & 29 & 19 & 32 & 44 & 22 & 2 & $<1$ \\
\hline $\begin{array}{l}\text { Engagement } \\
\text { Freizeit }\end{array}$ & 21 & 38 & 27 & 13 & 21 & 37 & 38 & 4 & 1 \\
\hline $\begin{array}{l}\text { Soziales, } \\
\text { polit. En- } \\
\text { gagement }\end{array}$ & 22 & 43 & 26 & 9 & 11 & 31 & 48 & 8 & 2 \\
\hline $\begin{array}{l}\text { Religiöses } \\
\text { Engage- } \\
\text { ment }\end{array}$ & 43 & 31 & 18 & 9 & 18 & 33 & 40 & 9 & 1 \\
\hline
\end{tabular}

Quelle: Österreichischer Wertebildungssurvey 2016 (ÖWBS), eigene Berechnungen 
Tab. 2 Logistische Regression für die Bewertung des Einflusses von Elternhaus, PartnerIn und biografischen Brüchen auf die Entstehung individueller Werthaltungen (Odds Ratios)

\begin{tabular}{|c|c|c|c|c|c|c|c|c|c|}
\hline \multirow{2}{*}{$\begin{array}{l}\text { Prägung durch } \\
\text { Lebensbereich } \\
\text { negativ }\end{array}$} & \multicolumn{3}{|c|}{ Elternhaus } & \multicolumn{3}{|l|}{ Partner } & \multicolumn{3}{|c|}{ Biografische Brüche } \\
\hline & $\operatorname{Exp}(B)$ & Sign. & SE & $\operatorname{Exp}(B)$ & Sign. & SE & $\operatorname{Exp}(B)$ & Sign. & SE \\
\hline $\begin{array}{l}\text { Frauen (Ref. } \\
\text { Männer) }\end{array}$ & 2,364 & $* * *$ & 0,130 & 2,477 & $* * *$ & 0,132 & 1,866 & $* * *$ & 0,174 \\
\hline \multicolumn{10}{|c|}{ Alter (Ref. 40-49 Jahre) } \\
\hline$>30$ Jahre & 0,604 & $*$ & 0,197 & 1,582 & $*$ & 0,198 & 0,788 & n.s. & 0,264 \\
\hline 30-39 Jahre & 0,957 & - & 0,196 & 1,141 & - & 0,201 & 1,101 & - & 0,284 \\
\hline 50-59 Jahre & 1,079 & - & 0,195 & 1,562 & $*$ & 0,197 & 0,810 & - & 0,258 \\
\hline +60 Jahre & 1,249 & - & 0,210 & 0,831 & - & 0,227 & 0,559 & $*$ & 0,265 \\
\hline \multicolumn{10}{|c|}{ Bildung (Ref. max. Pflichtschule) } \\
\hline $\begin{array}{l}\text { Lehre, BMS, } \\
\text { HaSch }\end{array}$ & 0,947 & - & 0,171 & 0,692 & $*$ & 0,173 & 0,915 & - & 0,231 \\
\hline $\begin{array}{l}\text { AHS, BMS, } \\
\text { Kolleg }\end{array}$ & 1,008 & - & 0,213 & 0,920 & - & 0,216 & 1,124 & - & 0,291 \\
\hline Uni, FH & 0,675 & - & 0,232 & 0,652 & - & 0,233 & 0,998 & - & 0,307 \\
\hline \multicolumn{10}{|c|}{ Migrationshintergrund im Vergleich zu ohne } \\
\hline Mit MH & 1,535 & $*$ & 0,166 & 1,080 & - & 0,177 & 0,821 & - & 0,217 \\
\hline \multicolumn{10}{|c|}{ Religiosität im Vergleich zu Mitte (4-6) } \\
\hline $\begin{array}{l}\text { Nicht religiös } \\
(1-3)\end{array}$ & 1,753 & $* * *$ & 0,150 & 1,346 & $*$ & 0,151 & 1,200 & - & 0,197 \\
\hline $\begin{array}{l}\text { Stark religiös } \\
(7-9)\end{array}$ & 1,067 & - & 0,200 & 1,203 & - & 0,198 & 0,542 & $* *$ & 0,229 \\
\hline \multicolumn{10}{|c|}{ Kontrollvariablen } \\
\hline Sozialraum & - & Inkl. & - & - & Inkl. & - & - & Inkl. & - \\
\hline Polit. Position & - & Inkl. & - & - & Inkl. & - & - & Inkl. & - \\
\hline Konstante & 0,323 & $* * *$ & 0,276 & 0,352 & $* * *$ & 0,285 & 3,574 & $* * *$ & 0,355 \\
\hline $\begin{array}{l}\text { Nagelkerke } R^{2} \\
\text { in } \%\end{array}$ & 9,6 & & & 10,5 & & & 7,0 & & \\
\hline$N$ & 1202 & & & 1122 & & & 1005 & & \\
\hline
\end{tabular}

Quelle: Österreichischer Wertebildungssurvey 2016 (ÖWBS), eigene Berechnungen

Signifikanzniveaus: $* p<0,05 ; * * p<0,01 ; * * * p<0,001$

sehen aber auch teils gute und teils negative Aspekte darin. Ein weiterer wichtiger Bereich sind Erfahrungen in der Ausbildung und im Berufsleben. Beinah $80 \%$ der ÖsterreicherInnen fühlen sich in ihren Werten durch ihre Arbeit (eher) stark geprägt. Ein Großteil davon bewertet die Prägung als überwiegend positiv. ${ }^{9}$ Lediglich die Schulausbildung bewerten auffallend viele Menschen (etwa 14\%) als eher oder sehr negativ prägend für ihre Werte. Über $40 \%$ berichten von gemischten Erfah-

\footnotetext{
$9 \mathrm{Da}$ in der Literatur immer wieder darauf hingewiesen wird, dass besonders im Arbeitsleben nicht nur intendierte Wertebildung, sondern verschiedene Erfahrungsebenen (Unternehmenskultur, Führungsstil, kollegiale Beziehungen) relevant sind (siehe auch Schein 2006), sollten auch hier in Zukunft die Einflüsse sich verändernder Arbeitsbedingungen (Digitalisierung, Prekarisierung) für die Werthaltungen in Österreich stärker berücksichtigt werden.
} 
rungen. Zudem ist in Bezug auf die Schul- und Ausbildung außerdem erstaunlich, dass trotz großen Bemühens von PädagogInnen, Wertebildung im Unterricht zu reflektieren (was sich u.a. in der Menge an Forschungsliteratur in diesem Bereich abbildet), dieser Bereich als vergleichsweise wenig prägend und erstaunlich negativ wahrgenommen wird. Ein vertiefender Blick auf die strukturelle Implementierung von Wertebildungsprozessen im österreichischen Bildungssystem wäre hier sicher lohnend (vgl. Bucher 2014; Feyerer 2015). Schließlich ist auch das Engagement in sozialen, politischen oder religiösen Gruppen für etwa ein Drittel der in Österreich lebenden Personen prägend. Auch diese Erfahrungen - von denen anzunehmen ist, dass sie größtenteils auf freiwilliger Basis geschehen - werden von einem großen Teil der in Österreich lebenden Personen als teils positiv, teils negativ bewertet; von ungefähr $10 \%$ sogar als überwiegend negativ. Auch hier ist erstaunlich, dass dieser von der Werteforschung immer wieder hervorgehobene und häufig selbstdeklarierte Ort der Entstehung individueller Werthaltungen (siehe z. B. Meyer-Ahlen 2007) von der österreichischen Bevölkerung als relativ unbedeutend eingestuft wird.

Zusammenfassend lässt sich mit Blick auf die Befunde in Tab. 1 festhalten, dass das familiäre Umfeld (Eltern, PartnerInnen), biografische Brüche und das Arbeitsleben besonders starke Einflüsse auf die Entstehung individueller Werthaltungen haben. Im Kontrast dazu haben jene Bereiche, in denen oft intendierte Wertebildung stattfindet (Schule, Ausbildung, Engagement in Organisationen) vergleichsweise wenig Relevanz.

Zusätzlich zeigen die Ergebnisse, dass der Einfluss von „,negativen“ Erfahrungen für die Entstehung individueller Werthaltungen größer ist, als dies auf Basis der bisherigen Forschungen zu vermuten war. In allen Lebensbereichen wird Prägung von zumindest einem Drittel der Befragten als teilweise negativ bewertet. Damit unterstreicht die quantitative Analyse die Befunde der Fokusgruppen und die Bedeutung von Abgrenzungen, Konflikten und biografischen Brüchen für die Entstehung individueller Werthaltungen. Vor allem im Übergang von einem in einen anderen Lebensbereich, etwa den von der Schule in die Arbeitswelt, erscheint die negative Bewertung von Wertebildungsprozessen plausibel, wenn man Sozialisationstheorien folgt. Edgar Schein $(1988,2006)$ etwa legt in seiner Forschung dar, dass der Eintritt in Organisationen und das Erlernen der dort geltenden Werte- und Normensysteme ein teilweise herausfordernder Prozess sein kann. „Organizations socialize their new members by creating a series of events which serve the function of undoing old values so that the person will be prepared to learn new values. This process of undoing or unfreezing is often unpleasant" (Schein 1988, S. 56). ${ }^{10}$ Eine weitere Erklärung für die Bedeutung negativer Prägungsmomente lässt sich mit Hans Joas' Konzept im Begriff der Selbsttranszendenz finden. Für Joas sind für die Entstehung individueller Werthaltungen Erfahrungen zentral, in denen der Wert einer Sache subjektiv evident ist und sie gleichzeitig aus ihrem bisherigen Selbst hinausgerissen werden. Er verweist auf Durkheims Konzept der „,kollektiven Ekstase“ und beschreibt Si-

\footnotetext{
10 In extremen Fällen, argumentiert Schein (1988), wird in Initiationsriten das alte Selbst symbolisch zerstört (z. B. Kleidungsvorschriften) und dem Individuum die Unterlegenheit bisheriger Werte vorgeführt. Dabei müssen Organisationen auch die Gegensozialisation von Peer-Groups verhindern, wenn diese nicht mit den Organisationswerten übereinstimmen.
} 
tuationen, in denen Menschen aus ihren geplanten Abläufen hinausgerissen werden, und gewisse zivilisatorische Regeln sowie die Selbstkontrolle wegfallen. Gleichzeitig verändert diese neue Situation alle bestehenden sozialen Beziehungen zwischen den Beteiligten (Joas 2006, S. 5-6). Schicksalsschläge können z. B. als solche Momente verstanden werden, in denen bestehende Strukturen plötzlich wegfallen und Beziehungen in emotional aufgeladenen Situationen neu verhandelt werden müssen. Sie können wertebildenden Charakter haben, da „die Dezentrierung der Person“ mit einer Bindung an die ,latenten propositionalen Gehalte der Erfahrungssituation“" einhergehen (Joas 2006, S. 8).

\subsubsection{Soziale Strukturiertheit von negativen Einflüssen auf die Entstehung individueller Werthaltungen}

Mithilfe der quantitativen Umfragedaten soll in einem abschließenden Schritt die soziale Strukturiertheit der Entstehung individueller Werthaltungen mittels logistischer Regressionsanalysen untersucht werden (z. B. nach Geschlecht, Bildungsstand, Religionszugehörigkeit). Für den vorliegenden Artikel werden wir uns auf die oben diskutierten Bereiche Familie (Elternhaus und Partnerschaft) sowie biografische Brüche als Beispiele beschränken.

Tab. 2 zeigt die Ergebnisse der Regressionsanalyse. Es wird ersichtlich, dass Frauen die Entstehung individueller Werthaltungen in den ausgewählten Bereichen negativer empfinden als Männer. Die starke Geschlechterspezifik dieser Erfahrung ist überraschend, da sie sich in den qualitativen Ergebnissen nicht in diesem Ausmaß angedeutet hat. In der internationalen Werteforschung sind Geschlechterunterschiede in der Wertepräferenz zwar nachgewiesen (beispielsweise ist Frauen Prosozialität/Humanismus wichtiger und Männern Macht), diese sind aber meist schwächer ausgeprägt als Alters- oder Länderunterschiede (Schwartz und Rubel 2005). Eine mögliche Erklärung für die vorliegenden Ergebnisse könnte sein, dass Frauen in Österreich noch immer stärker von ihrem Umfeld (Familie, Partnerschaft, Gemeinde) finanziell und sozial abhängig sind als Männer und daher von biografischen Brüchen und Konflikten in diesen Bereichen stärker betroffen werden (Bundeskanzleramt Österreich 2010). So bewerteten etwa 2008 in der letzten Europäischen Wertestudie mehr Frauen den Bereich Familie als wichtigsten Lebensbereich als Männer (Friesl et al. 2009, S. 127-128).

In Bezug auf das Alter zeigt sich, dass Unter-30-Jährige Prägung durch ihr Elternhaus besonders positiv bewerten. Die Prägung durch die eigene Partnerschaft bewerten Unter-30-Jährige sowie Menschen zwischen 50 und 60 Jahren negativer als andere Gruppen. Viele andere Bereiche werden von Über-60-Jährigen besonders positiv betrachtet, darunter auch biografische Brüche. Anzudeuten scheint sich hier eine Verschiebung der sozialen Eingebundenheit. Diese kann dazu beitragen, negative Konsequenzen von Schicksalsschlägen abzufedern und damit den Wertebildungsprozessen eine positive Konnotation zu geben. Während das Elternhaus für junge Erwachsene noch ein wichtiges Sicherheitsnetz bietet, ist das Leben später mehr vom Gelingen oder Nicht-Gelingen der Partnerschaft abhängig. Die ÖsterreicherInnen sind bis zum 30. Lebensjahr großteils ledig (53\%), während danach die Zahl der Verheirateten, aber auch Geschiedenen zunimmt. Entsprechend scheint es 
plausibel, dass Unter-30-Jährige (Partnersuche) sowie Über-50-Jährige (erwachsene Kinder, hohe Scheidungsrate) besonders häufig von negativer Prägung berichten. Der Wegfall von sozialen Beziehungen wird schließlich im Alter als natürlicher Teil des Lebens akzeptiert und nicht mehr so stark als außergewöhnliche Krise wahrgenommen. Zudem konnten die qualitativen Ergebnisse zeigen, dass biografische Brüche vor allem in der Rückschau ihre positiven Folgen für die Befragten offenbaren. Ältere Personen haben durch ihr Lebensalter eine höhere Wahrscheinlichkeit, bereits mit einigen Jahren Abstand auf Schicksalsschläge zurückblicken zu können.

Bei ÖsterreicherInnen mit und ohne Migrationshintergrund zeigen sich in den meisten Lebensbereichen keine signifikanten Unterschiede bezüglich negativer Prägungserfahrungen. Lediglich das Elternhaus stellt für MigrantInnen häufiger einen Ort negativer Werteprägung dar. Dies lässt sich besonders für MigrantInnen der zweiten Generation (in Österreich geboren) zeigen, welche zwischen den Werten der Eltern und der Aufnahmegesellschaft häufiger Spannungen erleben dürften (vgl. Bucher 2014; Ersanilli 2012; Hadjar et al. 2014). Zudem lässt sich festhalten, dass nicht-religiöse Personen scheinbar häufiger negative Prägungserfahrungen machen oder dieselben Erfahrungen negativer bewerten. Dies bildet sich besonders in Bezug auf das Elternhaus und die Partnerschaft ab. Personen, die angeben, sehr religiös zu sein, nehmen außerdem biografische Brüche häufiger positiv wahr. Der positive Effekt von Religiosität auf die Lebenszufriedenheit und Lebensbewältigung wird v. a. in der amerikanischen Forschung seit Langem kontrovers diskutiert (siehe z. B. Ellison et al. 1989; Hadaway 1978; Lim und Putnam 2010). Schließlich zeigen die Variablen Sozialraum und politische Selbstpositionierung keine signifikanten Unterschiede hinsichtlich negativer Prägungserfahrungen.

\section{Fazit}

Wie entstehen individuelle Werthaltungen? Wie gestaltet sich dieser Prozess genau und wodurch wird er beeinflusst? Der vorliegende Beitrag versucht diese Forschungslücken mit den Befunden eines Mixed-Methods-Projektes zu adressieren. Durch die Durchführung explorativer Fokusgruppen, die Entwicklung neuer quantitativer Skalen und deren Prüfung in einer repräsentativen Umfrage $(n=1500)$ zeichnet sich das Vorhaben gegenüber bestehender Werteforschung aus, welche bislang von theoretischen Debatten und standardisierten Surveys dominiert wird.

Die qualitativen Ergebnisse legen erstens nahe, dass individuelle Werthaltungen nicht nur durch intendierte Prozesse, z. B. in Institutionen wie Schulen, gebildet werden, sondern dass sich ihre Entstehung auf viele andere Lebensbereiche erstreckt. Als genauso zentral für Wertebildung erweist sich die Partnerschaft oder das Arbeitsleben. Zweitens entstehen individuelle Werthaltungen nicht nur in „sanften“ Lernund Vermittlungsprozessen. Die Befragten der Österreichischen Wertebildungsstudie streichen stattdessen plötzliche Umbrüche oder Konflikte als Erfahrungen heraus, in denen ihre Werte sich ändern, verfestigen oder erst ins Bewusstsein treten. Individuelle Werthaltungen scheinen sich hauptsächlich dann zu ändern, wenn Handlungsunsicherheit besteht, wenn Bekanntes hinterfragt und mit neuen Sichtweisen konfrontiert werden muss. Damit können wir an Erkenntnisse von Schubarth an- 
schließen, der in Zeiten gesellschaftlicher Veränderungen einen verstärkten Wunsch nach „klaren Wertorientierungen“ konstatiert (vgl. Schubarth 2010).

Diese Befunde werden drittens auch durch die quantitativen Ergebnisse bestätigt, die etwa die Wichtigkeit der Familie, der Arbeit und biografischer Brüche für die Entstehung individueller Werte klar abbilden. Erstaunlicherweise werden diese Lebensbereiche von den ÖsterreicherInnen wichtiger eingeschätzt, als typische Orte intendierter Wertevermittlung (Schule, religiöses und soziopolitisches Engagement). Viertens zeigen die Umfragedaten, dass negative Erfahrungen in allen Lebensbereichen einen bemerkenswert großen Anteil haben. Mehr als ein Drittel der Erlebnisse, die individuelle Werthaltungen prägen, werden von den TeilnehmerInnen als zumindest teilweise negativ bewertet. Schließlich konnte fünftens aufgezeigt werden, dass der soziale Kontext wertbildender Erfahrungen ein fruchtbares Thema für zukünftige Werteforschung ist. So wurde in den ausgewählten Lebensbereichen (Familie, Partnerschaft, biografische Brüche) deutlich, dass Frauen, MigrantInnen und nicht-religiöse Personen Wertebildungsprozesse vergleichsweise häufig als negativ wahrnehmen. Den Gründen und Mustern dieser sozialen Strukturiertheit von Werthaltungen und deren Entstehungsprozessen gilt es weiter nachzugehen.

Abschließend wollen wir an dieser Stelle einige Hinweise zum weiteren Forschungsbedarf formulieren, die sich auch aus den Limitationen unserer Studie ergeben. Erstens basieren unsere qualitativen und quantitativen Daten auf QuerschnittsErhebungen, können also keine Entwicklungen abbilden, sondern nur nachträgliche Reflexionsprozesse auf die Auswirkungen der Lebensführung einfangen. Als Ergänzung zu unseren Befunden und zu den Analysen, die mit komparativ angelegten Surveys möglich sind (z. B. Sozialer Survey Österreich, Europäische Wertestudie, European Social Survey), würde die Nutzung von Panelstudien oder qualitativen Langzeitstudien wichtige Ergänzungen zum Verständnis der Veränderungen individueller Werthaltungen im Lebensverlauf liefern (solche Daten liegen bisher in Österreich leider nicht vor). Zweitens wäre es produktiv, in anderen Primärerhebungen komplementäre Kriterien der Datengenerierung zu nutzen. Vorstellbar wäre zum Beispiel, bestehende Unterschiede - z. B. negativere Bewertung von Wertebildungsprozessen durch Frauen - durch weitere Feldphasen vertiefend zu untersuchen, und dabei das Geschlecht als Kriterium zur Fokusgruppenbildung zu priorisieren. Drittens erscheinen uns im vorliegenden Beitrag herausgearbeitete neue Befunde - z. B. der Einfluss von Konflikten und biografischen Brüchen auf Wertebildung - als eine Chance, interdisziplinäre Zusammenarbeit in der Werteforschung stärker zu forcieren (Ziebertz 2003). Bisher gibt es sowohl zwischen unterschiedlichen Disziplinen, aber auch zwischen theoretischen und empirischen Ansätzen nur wenig Austausch. Ein solcher Austausch könnte aus unserer Sicht zu einer vielgewünschten Konkretisierung des Wertebegriffs beitragen (z. B. Rohan 2000).

Funding Open access funding provided by University of Vienna.

Open Access Dieser Artikel wird unter der Creative Commons Namensnennung 4.0 International Lizenz (http://creativecommons.org/licenses/by/4.0/deed.de) veröffentlicht, welche die Nutzung, Vervielfältigung, Bearbeitung, Verbreitung und Wiedergabe in jeglichem Medium und Format erlaubt, sofern Sie den/die ursprünglichen Autor(en) und die Quelle ordnungsgemäß nennen, einen Link zur Creative Commons Lizenz beifügen und angeben, ob Änderungen vorgenommen wurden. 


\section{Literatur}

Bachleitner, Reinhard, und Wolfgang Aschauer. 2015. Unterwegs in der Freizeit. Zur Soziologie des Reisens. In Handbuch Freizeitsoziologie, Hrsg. Renate Freericks, Dieter Brinkmann, 325-352. Wiesbaden: VS.

Bertelsmann Stiftung. 2016. Werte lernen und leben. Theorie und Praxis der Wertebildung in Deutschland. Gütersloh: Bertelsmann.

Bohnsack, Ralf, und Aglaja Przyborski. 2006. Das Gruppendiskussionsverfahren in der Forschungspraxis. Opladen: Budrich.

Bohnsack, Ralf, Iris Nentwig-Gesemann, und Arnd-Michael Nohl. 2013. Die dokumentarische Methode und ihre Forschungspraxis. Berlin: Springer.

Bryman, Alan, und Emma Bell. 2015. Business research methods. Oxford: Oxford University Press.

Bucher, Anton. 2014. Der Ethikunterricht in Österreich: Politisch verschleppt - pädagogisch überfällig! Innsbruck: Tyrolia.

Bundeskanzleramt Österreich. 2010. Frauenbericht 2010. Bericht betreffend die Situation von Frauen in Österreich im Zeitraum von 1998 bis 2008. Wien, Bundesministerium für Frauen und Öffentlichen Dienst, Wien.

Castels, Stephen, Maja Korac, Ellie Vasta und Steven Vertovec. 2002. Integration: Mapping the Field. Report of a project carried out by the Center for Migration and Policy Research and Refugee Studies Centre, University of Oxford.

Creswell, John, und Vicki Plano Cark. 2011. Designing and conducting mixed methods research. London: SAGE.

Der Standard. 2016. Philosoph: „Ein erzwungener Wert ist niemals ein Wert“. [28.11.2016, 06:00]

Die Presse. 2015. Auf der Suche nach österreichischen Werten. [02.02.2015, 11:34]

Ellison, Christopher, David Gay, und Thomas Glass. 1989. Does religious commitment contribute to individual life satisfaction? Social Forces 68(1):100-123.

Ersanilli, Evelyn. 2012. Model(ling) citizens? Integration policies and value integration of Turkish immigrants and their descendants in Germany, France, and the Netherlands. Journal of Immigrant \& Refugee Studies 10(3):338-358.

Fend, Helmut, Fred Berger, und Urs Grob. 2009. Lebensverläufe, Lebensbewältigung, Lebensglück. Ergebnisse der LifE-Studie. Wiesbaden: VS.

Feyerer, Jakob. 2015. Der kompetente Bürger? Überlegungen zur Gestaltung Politischer Bildung in Österreich. SWS Rundschau 55(1):48-64.

Friesl, Christian, Ursula Hamachers-Zuba, und Regina Polak. 2009. Die Österreicher/innen: Wertewandel 1990-2008. Wien: Czernin.

GESIS, Leibniz-Institut für Sozialwissenschaften. 2014. Allgemeine Bevölkerungsumfrage der Sozialwissenschaften ALLBUS 2014. Köln, GESIS Datenarchiv.

Grusec, Joan, und Leon Kczynski. 1997. Parenting and children's internalization of values. A handbook of contemporary theory. New York: Wiley.

Hadaway, Christopher. 1978. Life satisfaction and religion: a reanalysis. Social Forces 57(2):636-643.

Hadjar, Andreas, Klaus Boehnke, Ariel Knafo, Ella Daniel, Anna-Lena Musiol, David Schiefer, und Anna Möllering. 2014. Intergenerationale Werteähnlichkeit, Distanz zu gesellschaftlichen MainstreamWerten und subjektives Wohlbefinden von MigrantInnen. In Zwischen den Generationen. Transmissionsprozesse in Familien mit Migrationshintergrund, Hrsg. Hilde Weiss, Philipp Schnell, und Gülay Ateş, 49-69. Wiesbaden: Springer.

Hoerning, Erika. 1987. Lebensereignisse: Übergänge im Lebenslauf. In Methoden der Biographie- und Lebenslaufforschung, Hrsg. Wolfgang Voges, 231-259. Wiesbaden: VS.

Joas, Hans. 1999. Die Entstehung der Werte. Mainz: Suhrkamp.

Joas, Hans. 2006. Wie entstehen Werte? Wertebildung und Wertevermittlung in pluralistischen Gesellschaften. Vortrag in der Reihe: Gute Werte, schlechte Werte. Gesellschaftliche Ethik und die Rolle der Medien. 15. September 2006, FSF. Berlin: Transkript. https://fsf.de/data/hefte/pdf/Veranstaltungen/ tv_impuls/2006_Ethik/Vortrag_Joas_authorisiert_061017.pdf. Zugegriffen: 24.08.2015.

Kohlberg, Lawrence. 1984. The psychology of moral development. The nature and validity of moral stages. Essays on moral development, Bd. 2. San Francisco: Harper \& Row.

Kohli, Martin. 1988. Normalbiographie und Individualität: Zur institutionellen Dynamik des gegenwärtigen Lebenslaufregimes. In Vom Ende des Individuums zur Individualität ohne Ende, Hrsg. HannsGeorg Brose, Bruno Hildebrand, 33-53. Opladen: Leske + Budrich. 
Kohn, Melvin, Kazimierz Slomczynski, und Carrie Schoenbach. 1986. Social stratification and the transmission of values in the family: a cross-national assessment. Sociological Forum 1(1):73-102.

Krone. 2015. Top-Anwalt sagt: „Unsere Werte sind für Zuwanderer uninteressant“. [15.05.2015, 15:25]

Lim, Chaeyoon, und Robert Putnam. 2010. Religion, social networks, and life satisfaction. American Sociological Review 75(6):914-933.

Lussi, Isabella, und Stephan Huber. 2015. Das Erleben von Anerkennung in der Schule und seine Relevanz für die Werteentwicklung von jungen Erwachsenen. Forum Qualitative Sozialforschung 16(3):60.

Meyer-Ahlen, Stefan H. 2007. Mehrwert Religion - Die besondere Bedeutung von Religion im Kontext der Werterziehung. In Braucht Werterziehung Religion?, Hrsg. Hans Joas, 67-100. Göttingen: Wallstein.

Morgan, David. 1997. Focus groups as qualitative research. Thousand Oaks: SAGE.

Nunner-Winkler, Gertrud, Marion Meyer-Nikele, und Doris Wohlrab. 2006. Integration durch Moral. Moralische Motivation und Ziviltugenden Jugendlicher. Wiesbaden: VS.

ÖWBS. 2016. Österreichischer Wertebildungssurvey, Institut für Soziologie, Universität Wien.

Piaget, Jean. 1932. Le jugement moral chez l'enfant. Paris: Presses Universitaires de France.

Polak, Regina. 2011. Grundlagenfragen und Situierung des Diskurses. In Zukunft. Werte. Europa. Die europäische Wertestudie 1990-2010: Österreich im Vergleich, Hrsg. Regina Polak, 23-62. Wien: Böhlau.

Polak, Regina, Christian Friesl, und Ursula Hamachers-Zuba. 2009. „Werte“ - Versuch einer Klärung. In Die Österreicher/innen. Wertewandel 1990-2008, Hrsg. Christian Friesl, Regina Polak, und Ursula Hamachers-Zuba, 13-34. Wien: Czernin.

Rohan, Meg J. 2000. A rose by any name? The values construct. Personality and social psychology review 4(3):255-277.

Schein, Edgar. 1988. Organizational socialization and the profession of management. Sloan Management Review 53(1):1-53.

Schein, Edgar. 2006. Organisationskultur. Bergisch Gladbach: Edition Humanistische Psychologie - EHP.

Scherr, Albert. 2013. Werte und Normen. In Soziologische Basics: Eine Einführung für pädagogische und soziale Berufe, Hrsg. Albert Scherr, 271-278. Wiesbaden: VS.

Schubarth, Wilfried. 2010. Die „Rückkehr der Werte“. Die neue Wertedebatte und die Chancen der Wertebildung. In Wilfried Schubarth, Karsten Speck und Heinz Lynen von Berg, Hrsg. Wertebildung in Jugendarbeit, Schule und Kommune, 21-41. Wiesbaden: VS.

Schubarth, Wilfried. 2016. Wertebildung in der Fachdebatte: Theoretische Grundlagen und pädagogische Konzepte. In Werte lernen und leben. Theorie und Praxis der Wertebildung in Deutschland, Hrsg. Bertelsmann Stiftung, 17-60. Gütersloh: Bertelsmann.

Schwartz, Shalom. 1994. Are there universal aspects in the structure and contents of human values. Journal of Social Issues 50(4):19-45.

Schwartz, Shalom, und Tammy Rubel. 2005. Sex differences in value priorities: cross-cultural and multimethod studies. Journal of Personality and Social Psychology 89(6):1010-1028.

Spates, James. 1983. The sociology of values. Annual Review of Sociology 9(1):27-49.

Standop, Jutta. 2005. Werte-Erziehung. Einführung in die wichtigsten Konzepte der Werteerziehung. Weinheim: Beltz.

Stein, Margit. 2012. Werteerziehung. In Handbuch Erziehung, Hrsg. Uwe Sandfuchs, Wolfgang Melzer, Bernd Dühlmeier, und Adly Rausch, 662-667. Bad Heilbrunn: Klinkhardt.

Stein, Margit. 2013. Wertetransmission als Aufgabe der Familie. In Werte und Wertebildung in Familien, Bildungsinstitutionen, Kooperationen. Beiträge aus Theorie und Praxis, Hrsg. Deutsches Rotes Kreuz e.V., Annegret Erbes, Charlotte Giese, und Heribert Rollik, 11-24. Berlin: Deutsches Rotes Kreuz.

Suri, Harsh. 2011. Purposeful sampling in qualitative research synthesis. Qualitative Research Journal 11(2):63-75

Thome, Helmut. 2003. Soziologische Wertforschung: Ein von Niklas Luhmann inspirierter Vorschlag für die engere Verknüpfung von Theorie und Empirie. Zeitschrift für Soziologie 32(1):4-28.

Vogel, Berthold. 2008. Biographical breaks, social inequality and political configuration. Perspectives of sociological unemployment research. Mittelweg 36(17:2):11-20.

Welzel, Christian. 2009. Werte- und Wertewandelforschung. In Politische Soziologie. Ein Studienbuch, Hrsg. Viktoria Kaina, Andrea Römmele, 109-139. Wiesbaden: VS.

Wiener Zeitung. 2017. Was sind Werte wert? [19.08.2017, 12:00]

Ziebertz, Hans-Georg. 2003. Religiöse Signaturen heute: ein religionspädagogischer Beitrag zur empirischen Jugendforschung. Gütersloh: Kaiser. 
Roland Verwiebe Univ. Prof. Dr.: Universitätsprofessor am Institut für Soziologie der Universität Wien. Forschungsinteressen: Soziale Ungleichheit, Arbeitsmarkt, Migration, Einstellungs- und Wertwandel.

Lena Seewann BA MA: Universitätsassistentin, Institut für Soziologie, Universität Wien. Forschungsinteressen: Einstellungs- und Wertwandel, Religion, Migration.

Margarita Wolf BA MA: Wissenschaftliche Projektmitarbeiterin, Institut für Soziologie, Universität Wien. Forschungsinteressen: Einstellungs- und Wertwandel, Nationalsozialismus in Deutschland und Österreich, Migration. 